wissen kompakt 2022 $16: 1-2$

https://doi.org/10.1007/s11838-021-00146-0

() The Author(s), under exclusive licence to Freier Verband Deutscher Zahnärzte (FVDZ) and Springer Medizin Verlag GmbH, ein Teil von Springer Nature 2022

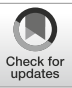

\title{
Frühkindliche Karies und Kreidezähne. Aktuelle Herausforderungen der Kinderzahnmedizin
}

\author{
K. Bekes' $\cdot$ J. M. Stein ${ }^{2,3}$ \\ ${ }^{1}$ Fachbereich Kinderzahnheilkunde, Universitätszahnklinik Wien, Medizinische Universität Wien, Wien, \\ Österreich \\ ${ }^{2}$ Klinik für Zahnerhaltung, Parodontologie und Präventive Zahnheilkunde, Universitätsklinikum RWTH \\ Aachen, Aachen, Deutschland \\ ${ }^{3}$ Praxiszentrum für Implantologie, Parodontologie und Prothetik, Aachen, Deutschland
}

Liebe Kolleginnen und Kollegen,

mit der ersten wissen-kompakt-Ausgabe dieses Jahres möchten wir die Gelegenheit nutzen, nach längerer Zeit wieder einmal den Fokus auf die Kinderzahnheilkunde zu legen. Dabei sollen insbesondere zwei Krankheitsbilder in den Vordergrund gestellt werden, die die Kinderzahnmedizin derzeit besonders fordern: Eines dieser Aufgabengebiete ist nicht neu, denn trotz der Präventionserfolge in den letzten Jahren stellt die frühkindliche Karies („early childhood caries") nach wie vor eine entscheidende Herausforderung und nicht gelöste Problematik dar. Kennzeichnend für die Erkrankung ist eine Glattflächenkaries mit schnell fortschreitenden Läsionen, die schon kurz nach Durchbruch der ersten Milchzähne beginnt. Aktuelle Daten aus den epidemiologischen Begleituntersuchungen zur Gruppenprophylaxe der Deutschen Arbeitsgemeinschaft für Jugendzahnpflege belegen, dass knapp 14\% der 3-jährigen Kinder, die eine Kindertagesstätte besuchen, bereits eine Karies aufweist. Im Durchschnitt sind bei diesen Kindern 3,57 Zähne betroffen. Hinzu kommt ein sehr geringer Sanierungsgrad, denn $73 \%$ der kariösen Zähne sind nicht behandelt worden.

Zudem werden in der Praxis zunehmend Kinder mit Kreidezähnen (MolarenInzisiven-Hypomineralisation, MIH) vorstellig, deren Zähne sich klinisch durch Porositäten mit wiederkehrenden Schmelz- frakturen in Kombination mit einer mitunter starken Hypersensibilität sowie sich wiederholenden Füllungsverlusten auszeichnen. Am Anfang noch als Zufallsbefund abgetan, hat diese Erkrankung des Zahnschmelzes inzwischen große klinische Relevanz erreicht. Neben der Karies zählt die MIH zu den häufigsten Erkrankungen der Zähne im Kindes- und Jugendalter. Nach der aktuellen Deutschen Mundgesundheitsstudie sind $28,7 \%$ der 12-Jährigen betroffen.

》) Neben der Karies zählt die MIH zu den häufigsten Erkrankungen der Zähne im Kindes- und Jugendalter

Inhaltlich finden Sie in dieser Ausgabe vier Beiträge, die sich mit den angesprochenen Themen auseinandersetzen. Prof. Schiffner gibt einen Einblick in die Epidemiologie der frühkindlichen Karies und stellt aktuelle Präventionsansätze für die Praxis dar. In detaillierter Weise erläutert er, wie mit einer frühzeitig einsetzenden zahnärztlichen Betreuung und aktualisierten Fluoridierungsempfehlungen kürzlich verbesserte Präventionselemente für Kleinkinder geschaffen wurden.

Prof. Krämer widmet sich in seinem Artikel den restaurativen Möglichkeiten des Milchzahnerhalts und den damit einhergehenden besonderen Anforderungen.

Prof. Kühnisch beleuchtet rezente Möglichkeiten der Endodontie im Milchgebiss und legt dabei den Fokus auf die Caries- 


\section{Editorial}

profunda-Therapie mit der selektiven Kariesexkavation und die Pulpotomie.

Abschließend gibt Frau Prof. Bekes in ihrem Übersichtsbeitrag zur MIH einen Einblick in das klinische Erscheinungsbild der Erkrankung, die derzeit diskutierten ätiologischen Faktoren, die Möglichkeiten der Diagnostik sowie rezente Therapiemethoden.

Liebe Kolleginnen und Kollegen, wir wünschen Ihnen viel Freude bei der Lektüre dieser Ausgabe.

Auf gesunde Zähne von Anfang an ein Leben lang!

Herzlichst

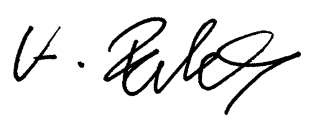

Prof. Dr. Katrin Bekes, MME

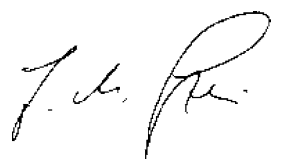

Prof. Dr. Jamal M. Stein, MSc.

\section{Korrespondenzadresse}

\section{Prof. Dr. K. Bekes, MME}

Fachbereich Kinderzahnheilkunde, Universitätszahnklinik Wien, Medizinische Universität Wien

Sensengasse 2a, 1090 Wien, Österreich

katrin.bekes@meduniwien.ac.at

\section{Prof. Dr. J. M. Stein, MSc}

Klinik für Zahnerhaltung, Parodontologie und Präventive Zahnheilkunde, Universitätsklinikum RWTH Aachen

Pauwelsstraße 30, 52074 Aachen, Deutschland jstein@ukaachen.de

Interessenkonflikt. K. Bekes und J.M. Stein geben an, dass kein Interessenkonflikt besteht. 